\title{
Optimum Radiological Screening Examination for Lumbar Spine
}

Roya Etemad-Rezai (1), B.Sc. and Paul V. Fenton (2), M.D.,F.R.C.P.(C)

To whom correspondence should be addressed:

1. Radiology Department

Kingston General Hospital, ON, Canada K7L 2V7

\section{ABSTRACT}

A retrospective study was performed to assess the diagnostic contribution of oblique view films of the lumbar spine, to information obtained from anteroposterior (AP) and lateral films, as an initial screening tool for the detection of pars interarticularis defects. Twenty-two cases of lumbar spondylolysis were selected from 243 lumbar spine reports, randomly combined with 40 plain X-rays of normal lumbar spines, and evaluated by radiology residents. The frequency of correctly detecting a pars defect on lateral vs. right and left oblique views was determined. Of the bilateral spondylolyses, $85 \%$ were diagnosed on lateral films compared to $35 \%$ on oblique radiographs. Both views gave poor diagnostic yield in detecting unilateral pars defects. In evaluating a total of $186 \mathrm{X}$-rays, an average of 31 oblique films were incorrectly diagnosed, as compared to an average of 14 misdiagnosed lateral films. Considering the low sensitivity associated with the use of oblique view radiography, in addition to the extra cost and significantly increased radiation exposure seen with this procedure, our findings indicate that oblique views should be used only for selected patients who might require further investigation. We therefore recommend that the initial lumbosacral radiological evaluation be limited to AP and lateral views.

\section{INTRODUCTION}

"Low back pain is one of the most common and incapacitating disorders in the modern society and the second leading cause of industrial absenteeism, behind only the common cold" (1). The assessment of patients with persistent low back pain includes a thorough history and physical examination, often followed by plain X-rays of the lumbosacral spine. In most general hospitals, the typical radiological evaluation of the lumbosacral spine in both adult and pediatric patients with back pain consists of five views: anteroposterior (AP), lateral, right and left obliques, and L5-S1 spot lateral (2).

The skin, bone marrow, and gonads are subject to the highest radiation exposure rate during a radiological examination of the lumbosacral column. The dose of radiation exposure to the gonads is particularly higher in oblique view radiography than from either AP or lateral views (3). Cost-effectiveness, degree of radiation 
exposure, and optimum standards of diagnostic sensitivity are the major factors to consider when designing or determining a reasonable screening examination in diagnostic radiology. Therefore, it is not surprising that the optimum number of views for an adequate plain film evaluation remains controversial (2-6).

Among disorders manifesting low back pain, lumbar spondylolysis, a common bony defect of the pars interarticularis of the lumbar spine, is considered to have the highest requirement for oblique view radiography. The defect is usually visible on a lateral X-ray of the lumbosacral junction (7) (Fig. 1). Currently, oblique views are considered the gold standard for diagnosing pars interarticularis defects in current clinical radiological practice. In diagnosing lumbar spondylolysis on an oblique film, one can take advantage of the resemblance of the outlines of the posterior elements to a Scotch terrier, first described as "Scotty dog" by Lachapele (Fig. 2). The ear of the dog is the superior articular process, the nose is the transverse process, the eye is the pedicle, and the front leg is the inferior articular process (7). A typical isthmic spondylolysis is seen as a lucency crossing the pars interarticularis at the neck of the Scotty dog.

While low back pain is the predominant complaint associated with this condition, other major symptoms include gait abnormalities, postural deformities, and hamstring tightness (7). Many theories have been suggested to explain the etiology of spondylolysis including congenital, developmental, degenerative, pathological, and traumatic causes (8). The idea of a purely congenital etiology has been dismissed since spondylolysis has never been detected in the fetus or the newborn. However, the incidence of pars defects in the adolescent population, the majority of whom are asymptomatic, is estimated to be $6 \%$ (7).

Back pain is a common cause of visits to orthopedic surgeons and emergency departments. These physicians are often the first to order plain X-rays as the initial step in assessing patients with low back pain. Therefore, their diagnoses, decisions on management, and prognoses of these patients depend on or rely on the usefulness and diagnostic yield of the screening exam.

Considering the high radiation exposure, extra cost, and low diagnostic yield of oblique lumbar X-rays that have been described in current literature (2-4), the purpose of this study is to determine the usefulness of oblique views, as a diagnostic adjunct to AP and lateral views, in screening for low back pain. We conducted a retrospective study to determine the frequency of detecting a pars defect in a random combination of oblique and lateral lumbar series. Since our goal was to determine the usefulness of oblique views to nonradiologists as a diagnostic tool for low back pain, we used eight radiology residents, from all four years of residency training, to simulate a group of physicians with limited radiological experience, acting upon the interpretation of a screening exam.

\section{METHODS}

A computer printout of 369 patient charts from March 1990 to March 1994 was obtained from the medical record offices of the Kingston General Hospital $(\mathrm{KGH})$ and Hotel Dieu Hospital. These charts were selected under the following diagnostic description codes: anomalies of spine, nonspecific back disorder, lumbosacral spondylolysis, acquired spondylolisthesis, lumbago, spinal stenosis, and ankylosing spondylitis.

After reviewing their imaging history, 243 patients were found to have had a radiological examination. To eliminate potential confounding variables, 57 patients who had undergone lumbar CT-scans, MRI, bone scans, myelograms, tomograms, or had received surgical hardware such as fixation plates, pedicle screws, or metal wires were eliminated from the study.

Of the remaining 186 patients with plain lumbar radiographs, 22 were diagnosed and reported to have had unilateral and bilateral pars interarticularis defects in their lumbar spine. These diagnoses were based on the evaluation of four views (AP, lateral, and two obliques) by licensed staff radiologists of the KGH. The oblique and lateral films of 22 spondylolysis cases were subsequently examined by an experienced 
musculoskeletal radiologist to confirm the presence of a lytic pars defect.

The 22 films were then randomly mixed with oblique and lateral films of 40 control cases (no pars defect) and presented to eight radiology residents of the KGH (one first-year, four second-year, two third-year, and one fourth-year) for examination. In each case, the right and left oblique views were shown together while the lateral views were presented individually. A grading system of "present" or "not present" was used to assess the existence or absence of a pars defect. In addition, the residents were asked to distinguish between a unilateral and a bilateral spondylolysis.

\section{RESULTS}

Of 186 patients with lumbosacral spine complaints, 22 were diagnosed with lumbar spondylolysis. This represents an $11.8 \%$ prevalence of the pars defect among the patient population studied. In 21 cases (95\%), the defect occurred at the level of L5; in one case, the defect involved an accessory lumbar vertebral body, L6. Bilateral spondylolysis was reported in 20 of the cases $(91 \%)$. The only unilateral spondylolyses were one left-sided and one right-sided pars defect (9\%). Eleven patients diagnosed with spondylolysis also had an L5 on S1 spondylolisthesis ranging from minimal forward slip to grade IV anterolisthesis.

The number of oblique and lateral films used in this study to detect normal, unilateral, and bilateral spondylolysis is shown in Table 1. The detection rates of total, bilateral, and unilateral pars defects using each radiographic view are summarized in Tables 2, 3, and 4, respectively. Briefly, bilateral pars defects were more often correctly diagnosed on lateral view radiographs (85\%), compared with oblique view radiographs (35\%). In contrast, a higher percentage of normal lumbar spines was detected on oblique view radiographs $(90 \%)$, compared to lateral films $(77.5 \%)$. The ability to detect a unilateral pars defect was poor with both the lateral $(0 \%)$ and oblique views (6.25\%). Finally, Table 5 summarizes the specificity and sensitivity of both radiographic views to diagnose bilateral pars defects.

\section{DISCUSSION}

Current radiological practice advocates the use of oblique views to diagnose pars interarticularis defects. However, the specific advantage of using this technique, which is associated with excess costs and high radiation exposure to the gonadal region is not fully known. In our study $85 \%$ of the bilateral pars defects were missed on the oblique view whereas only $15 \%$ were missed on the lateral view. Possible explanations for this finding may include the following: (i) To see a proper isthmus profile on an oblique film, the patient must be positioned at an optimum oblique angle. From a practical point of view, it is far easier to find a perfect lateral position than to find the optimum oblique position. (ii) In general, anatomic visualization and three-dimensional interpretation of an oblique lumbar spine radiograph might be more complicated than a lateral lumbar spine radiograph. This may be due to superimposing elements such as the iliac crest or the presence of bowel gas which could be mistaken for a defect. Lack of experience and familiarity with oblique films could also lead to lower rates of accurate diagnoses. (iii) Furthermore, 11 cases of pars defects were accompanied by L5 on S1 spondylolisthesis. Anterior subluxation, the forward slip of the L5 vertebral body, is a subtle change but can be used as an important clue for the presence of a pars defect. However, this sign may be difficult to evaluate on the oblique film.

Unilateral pars defects were poorly diagnosed using either the lateral or the oblique view. However, the significance of this finding is questionable due to the small sample size of only two unilateral cases.

The only time oblique views were shown to have had a higher detection rate than lateral films was in radiographs of normal patients. For oblique views, $90 \%$ correctly detected a normal case, compared to $77.5 \%$ of lateral views in the same category. 
Overall, lateral films were found to be more sensitive than oblique films, indicating their superiority in correctly diagnosing patients with disease. In contrast, oblique films were found to have a higher specificity than lateral view films indicating the advantage of oblique films in detecting those patients without disease (Table 5).

Evaluation of a total of 186 lumbar radiographs resulted in an average of 45 incorrect diagnoses (ranging from 34 to 54). Fourteen of the misdiagnosed films were lateral views whereas 31 were oblique views. This finding may indicate that the radiology residents had more difficulty reading oblique radiographs than lateral ones. Furthermore, non-radiologists, with similarly limited expertise and experience in assessing X-rays, may face the same problem.

The major finding of this study is that lateral view radiographs may be more effective than oblique views in detecting bilateral pars defects. In addition, limitations in detecting unilateral pars defects were encountered when using either technique. The main problems are distinguishing unilateral from bilateral pars defects, and identifying which side a given unilateral defect occurs. The significance of such a limitation is debatable considering the rare occurrence and low therapeutic yield of managing a unilateral spondylolysis.

Oblique views, in addition to being expensive, have previously been shown to add significantly to the gonadal dosage of radiation. Therefore, considering their low sensitivity in detecting lumbar spondylolysis, we recommend that the initial radiographic examination of the lumbosacral spine in patients presenting with low back pain be limited to AP and lateral views. Oblique angle views should only be used in cases where an abnormality cannot be ruled out on the basis of a standard two-view examination, or for further investigation if symptoms persist.

\section{ACKNOWLEDGEMENTS}

We wish to thank the radiology departments of Kingston General Hospital and Hotel Dieu Hospital for the use of their X-rays and patient data base, and radiology residents of $\mathrm{KGH}$ for taking part in our diagnostic trial.

\section{REFERENCES}

1. Giles L. Anatomical basis of low back pain. Baltimore: Williams \& Wilkins, 1989:1.

2. Roberts F, Kishore P, Cunningham M. Routine oblique radiography of the pediatric lumbar spine: is it necessary? American Journal of Roentgenology 1978;131:297-298.

3. Gehweiler J, Daffner R. Low back pain: the controversy of radiologic evaluation. American Journal of Roentgenology 1982;140:109-112.

4. Scavone J, Latshaw R, Weidner W. Anteroposterior and lateral radiographs: an adequate lumbar spine examination. American Journal of Roentgenology 1981;136:715-717.

5. Eisenberg R, Hedgehock M, William E, et al. Optimum radiologic examination for consideration of compensation awards: cervical and lumbar spines. American Journal of Roentgenology

1980;135:1071-1074.

6. Lisbon E, Bloom R, Dinari G. Oblique lumbar spine radiographs: importance in young patients. Radiology 1984;151:89-90.

7. Hardy RW. Lumbar Disk Disease, 2nd edition. New York: Raven Press, Ltd., 1993;35,213. 
8. Cope R. Acute traumatic spondylolysis: report of a case and literature review. Clinical Orthopedics and Related Research 1988;230:162-165.

\section{AUTHOR BIOGRAPHY}

Roya Etemad-Rezai received a B.Sc. in biochemistry and German from Concordia University (Montreal, Quebec) in 1992. She is presently a fourth-year medical student at Queen's University (Kingston, Ontario). His work on Lumbar Spondylolysis was conducted during her second year of medical education in the Radiology Department, Kingston General Hospital. His current research interests are in the field of neuroradiology. 